\title{
Visfatin/PBEF/Nampt: a new cardiovascular target?
}

\author{
1 Department of Pharmacology and Therapeutics, Universidad Autónoma de Madrid, Madrid, Spain \\ 2 Department of Medicine, Universidad Autónoma de Madrid, Madrid, Spain \\ ${ }^{3}$ Obesity Unit, Service of Endocrinology, Hospital Universitario de La Princesa, Madrid, Spain
}

Concepción Peiró ${ }^{1 *}$, Tania Romacho ${ }^{1}$, Raffaele Carraro ${ }^{2,3}$ and Carlos F Sánchez-Ferrer ${ }^{1}$

\author{
Edited by: \\ Y.W. Kwan, Chinese University of \\ Hong Kong, Hong Kong \\ Reviewed by: \\ Marilyn J. Cipolla, University of \\ Vermont, USA \\ Michael A. Hill, University of Missouri, \\ USA \\ Eric Thorin, Montreal Heart Institute, \\ Canada

\section{*Correspondence:} \\ Concepción Peiró, Departamento de \\ Farmacología y Terapéutica, Facultad \\ de Medicina, Universidad Autónoma \\ de Madrid, Arzobispo Morcillo, 4, \\ 28029 Madrid, Spain. \\ e-mail:concha.peiro@uam.es
}

\begin{abstract}
In the last years, a growing interest has emerged toward understanding the role of adipocytokines in the development of cardio-metabolic complications. Five years ago, visfatin/PBEF/Nampt was identified as a novel adipocytokine. In the context of metabolic disorders, extracellular visfatin/ PBEF/Nampt was initially claimed as a potentially beneficial molecule due to its insulin-mimetic and glucose-lowering properties. Nevertheless, growing evidence has since then unveiled that visfatin/PBEF/Nampt may rather be a biomarker of inflammation and endothelial damage, and also a direct regulator of the cardiovascular system that modulates cell proliferation and survival, extracellular matrix, vascular reactivity, and inflammation. On one side, the blockade of the deleterious cardiovascular actions of visfatin/PBEF/Nampt is being regarded as a potential approach to prevent and treat, not only cardio-metabolic complications, but also other pathologies implying excessive angiogenesis. Conversely, the administration of visfatin/PBEF/Nampt has shown beneficial effects in different ischemic conditions. Further research is required to evaluate the real value of visfatin/PBEF/Nampt as a pharmacological target.
\end{abstract}

Keywords: visfatin/PBEF/Nampt, adipocytokine, inflammation, proliferation, atherosclerosis

\section{INTRODUCTION}

The adipose tissue is nowadays considered as an active endocrine organ that produces a heterogeneous series of bioactive factors, the so-called adipocytokines. These adipocytokines can act locally within the adipose tissue, but they can also reach distant organs through the systemic circulation, where they can exert a wide range of biological actions, including the regulation of food intake and body weight, insulin sensitivity, reproduction, immunity, inflammation, or vascular homeostasis (Lau et al., 2005; Guzik et al., 2006). Importantly, an imbalanced adipocytokine production, as observed in clinical metabolic conditions including obesity and type 2 diabetes mellitus, has been associated with adipose tissue inflammation and the pathogenesis of insulin resistance, but also with chronic systemic inflammation and endothelial dysfunction (Karastergiou and Mohamed-Ali, 2010). In the last years, a growing interest has emerged to understand the involvement of adipocytokines as direct players in the development of cardiovascular complications associated to metabolic diseases.

\section{VISFATIN/PBEF/NAMPT}

In 2005, Fukuhara et al. (2005) first identified visfatin as an adipocytokine. The authors also described visfatin as an insulin-mimetic with potential beneficial effects in clinical conditions, such as diabetes mellitus. However, as the glucose-lowering properties of visfatin were hardly reproduced, the authors later on retracted from such statement (Fukuhara et al., 2007). Visfatin was found to be identical to pre-B cell colony-enhancing factor (PBEF), a 52-kDa cytokine acting on early B-lineage precursor cells (Samal et al., 1994) and also to the enzyme nicotinamide phosphoribosyltransferase (Nampt) (Rongvaux et al., 2002). In vertebrates, intracellular Nampt plays an essential role in the biosynthesis of NAD+ by converting nicotinamide into nicotinamide mononucleotide (NMN), which is in turn transformed into NAD+ by nicotinamide/nicotinic acid mononucleotide adenylyltransferase (Nmnat) (Formentini et al., 2009) (Figure 1). Based on these observations, visfatin is nowadays termed as visfatin/PBEF/Nampt.

Two different forms of visfatin/PBEF/Nampt have been identified to date. On one side, intracellular visfatin/PBEF/Nampt plays a central role in maintaining the activity of NAD-dependent enzymes and is implicated in the regulation of cellular metabolism in response to nutrient availability, maturation and survival (Revollo et al., 2004; van der Veer et al., 2005, 2007; Ho et al., 2009). On the other side, extracellular visfatin/PBEF/Nampt can be synthesized and released to the extracellular milieu, not only by adipocytes but also by many different cell types, where it can exert a wide range of actions in a paracrine or endocrine manner. Indeed, extracellular visfatin/PBEF/Nampt shows a slightly higher molecular weight than the intracellular isoform and seems to undergo post-translational modifications (Revollo et al., 2004, 2007).

In the context of metabolic diseases, circulating visfatin/PBEF/ Nampt levels have been found elevated in different clinical conditions, such as obesity, type 2 diabetes mellitus and the metabolic syndrome (Fukuhara et al., 2005; Sandeep et al., 2007). However, as recently reviewed, there is some controversy among the different reports studying the variation of visfatin/PBEF/Nampt levels in these disease states, where visfatin/PBEF/Nampt has also been found unmodified or even lower compared to healthy controls (Filippatos et al., 2010).

Although visfatin/PBEF/Nampt was initially claimed as being preferentially released by visceral versus subcutaneous fat (Fukuhara et al., 2005), this latter affirmation remains controversial (Arner, 2006). Importantly, visfatin/PBEF/Nampt has been detected in the 


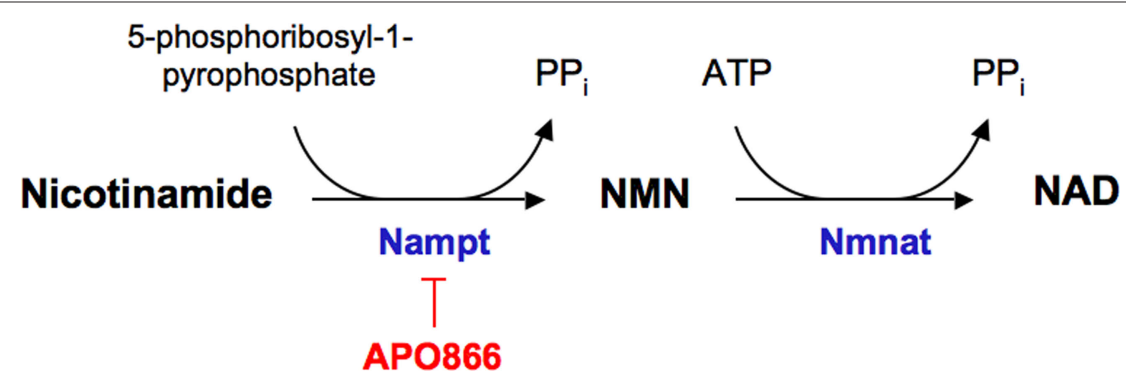

FIGURE 1 | Nicotinamide phosphoribosyltransferase (Nampt) enzymatic activity. Nampt uses nicotinamide as substrate to yield nicotinamide mononucleotide (NMN). In a subsequent step, NMN can be transformed into nicotinamide adenine dinucleotide (NAD+) by nicotinamide mononucleotide adenylyltransferase (Nmnat). The experimental drug AP0866 inhibits Nampt activity. ATP, adenosine triphosphate. perivascular fat of vessels, such as aorta or coronary artery, in both animal models and humans (Wang et al., 2009; Spiroglou et al., 2010). In fact, visfatin/PBEF/Nampt is released by a wide series of cell types, including activated monocytes/macrophages that can directly interact with vascular cells (Stephens and Vidal-Puig, 2006). These findings indicate that, besides circulating visfatin/ $\mathrm{PBEF} / \mathrm{Nampt}$, locally synthesized visfatin/PBEF/Nampt may also exert a relevant paracrine action in regulating the vascular function. This review will focus on the actions elicited by extracellular visfatin/Nampt/PBEF on the cardiovascular system (summarized in Figure 2), where it might become a novel therapeutic target.

\section{VASCULAR CELL PROLIFERATION ENDOTHELIAL CELLS}

In the last years, visfatin/PBEF/Nampt has been identified as a pro-angiogenic molecule. Hence, visfatin/PBEF/Nampt concentration-dependently promotes cell proliferation, migration and capillary-like tube formation in human umbilical vein endothelial cells cultures (HUVEC) (Kim et al., 2007; Adya et al., 2008a; Lovren et al., 2009; Xiao et al., 2009). In vivo, visfatin/PBEF/Nampt promotes the formation of functional neovessels (Kim et al., 2007). Furthermore, the injection of a plasmid containing visfatin/PBEF/ Nampt in a mouse model of unilateral limb ischemia results in improved limb perfusion as compared to untreated animals (Lovren et al., 2009).

The proliferative effect of visfatin/PBEF/Nampt in endothelial cells relies, at least in part, on the synthesis and secretion of vascular endothelial cell growth factor (VEGF) (Adya et al., 2008a), a key molecule in endothelial proliferation and neovessel formation. Visfatin/PBEF/Nampt also up-regulates the VEGF receptor 2, which mediates the angiogenic actions of VEGF (Adya et al., $2008 \mathrm{a}, \mathrm{b})$. An enhanced production of nitric oxide (NO), as the consequence of increased expression and activity of endothelial nitric oxide synthase (eNOS), has also been proposed to be on the basis of the pro-angiogenic action of visfatin/PBEF/Nampt in human endothelial cells (Lovren et al., 2009). Furthermore, visfatin/PBEF/Nampt activates dimethylarginine dimethylaminohydrolase (DDAH) (Xiao et al., 2009), an enzyme that hydrolyses the eNOS inhibitor L-arginine analog asymmetric dimethylarginine (ADMA), and upregulates VEGF expression and release in endothelial cells (Fiedler et al., 2009). Furthermore, visfatin/ PBEF/Nampt enhances the expression, protein levels and activity of matrix metalloproteinases (MMP)-2/9, as pivotal enzymes that facilitate angiogenesis through the degradation of the extracellular matrix, while it decreases the levels of the tissue inhibitors of MMP (TIMP)-1 and 2 (Adya et al., 2008a). Finally, both monocyte chemottractant protein (MCP)-1 (Adya et al., 2009) and fibroblast growth factor (FGF)-2 (Bae et al., 2009) have also been identified as mediators of visfatin/PBEF/Nampt-induced angiogenesis.

Regarding intracellular signaling, several reports have shown that phosphatidylinositol 3-kinase (PI3K)/Akt and the mitogenactivated protein kinases (MAPK) extracellular signal-regulated kinase (ERK) 1/2 mediate most of the cellular events leading to visfatin/PBEF/Nampt-induced endothelial cell proliferation (Kim et al., 2007; Adya et al., 2008a, 2009; Bae et al., 2009). Other studies have involved the activation of the transcription factors nuclear factor (NF)- $\kappa \mathrm{B}$ (Adya et al., 2009) and signal transducer and activator of transcription 3 (STAT3), the latter favoring the release of interleukin (IL)-6 (Kim et al., 2009). Figure 3 depicts the main cellular events triggered by visfatin/PBEF/Nampt leading to endothelial cell proliferation and migration.

Based on its pro-angiogenic properties, visfatin/PBEF/Nampt has been proposed as a potential therapeutic agent to improve blood supply in certain pathological conditions characterized by restricted blood flow such as macrovascular peripheral limb ischemia. On the other hand, visfatin/PBEF/Nampt might also be a promoter of dysregulated angiogenesis that is involved in clinical conditions such as ischemic heart disease, diabetes, or atherosclerosis and may therefore arise as a novel pharmacological target for treating such conditions. In addition, a growing interest is emerging toward visfatin/Nampt/PBEF as a therapeutic target for cancer treatment (Garten et al., 2009; Bi and Che, 2010). By inhibiting Nampt activity, the formation of new vessels that can support tumor growth might be restricted. In this context, two Nampt inhibitors FK866 (or APO866) and CSH-828 are being used in clinical trials (Ravaud et al., 2005; Pogrebniak et al., 2006).

\section{VASCULAR SMOOTH MUSCLE CELLS}

Vascular smooth muscle cell proliferation is a hallmark of the development of atherosclerotic lesions. Besides endothelial cells, visfatin/PBEF/Nampt can also act as a growth factor for vascular smooth muscle cells. Indeed, Wang et al. (2009) have shown that visfatin/PBEF/Nampt contained in the perivascular fat promotes DNA synthesis and cell proliferation in rat aortic smooth muscle 


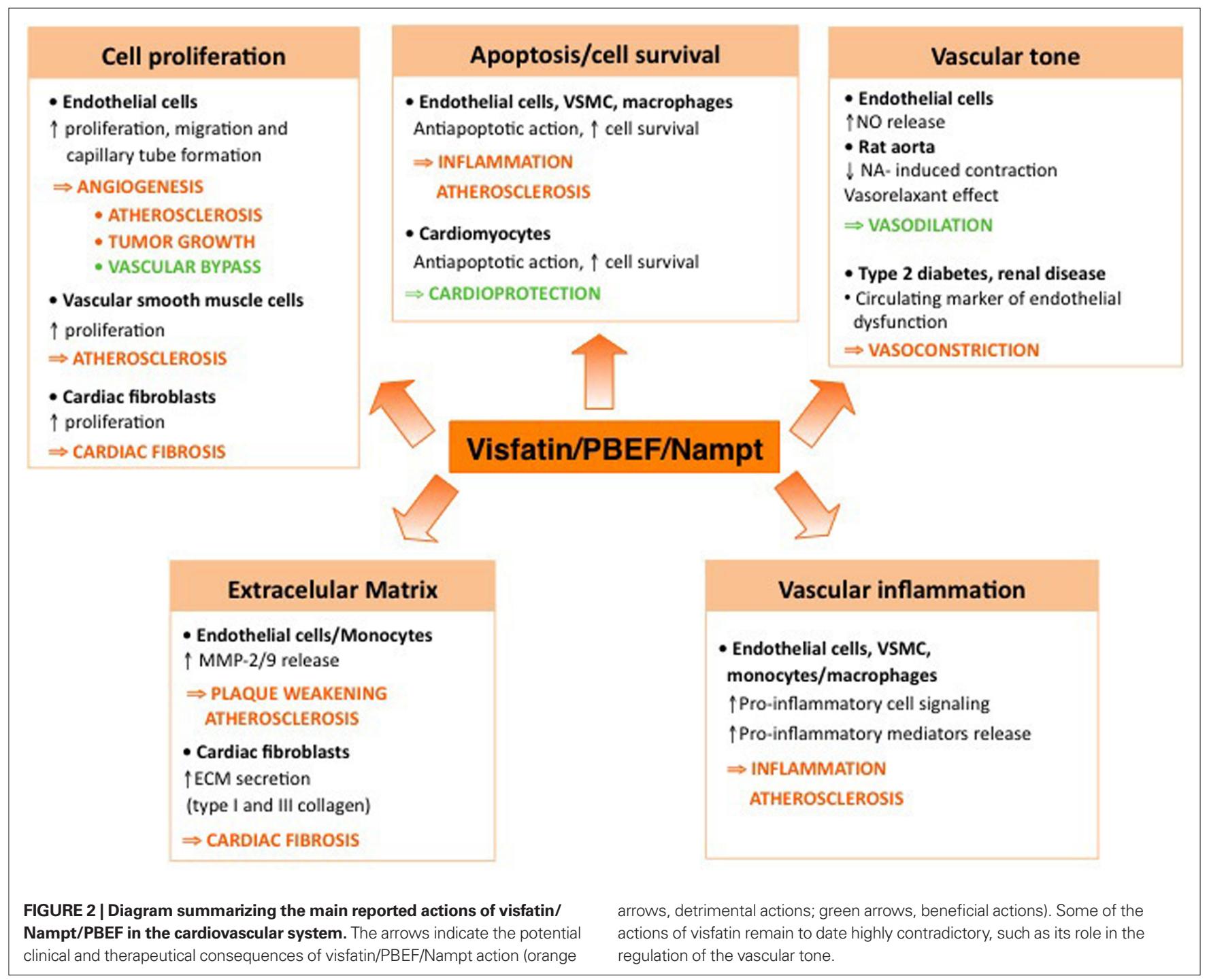

cell cultures through Nampt enzymatic activity. Hence, the proliferative action of visfatin/PBEF/Nampt is mimicked by the final product of the Nampt reaction, NMN, and is abolished by the Nampt pharmacological blocker FK866 (or APO866) (Wang et al., 2009). Intracellularly, the activation of ERK $1 / 2$ and p38 MAPK mediates visfatin/PBEF/Nampt-induced proliferation in vascular smooth muscle cultures (Wang et al., 2009). Through its growthpromoting properties for vascular smooth muscle, visfatin/PBEF/ Nampt emerges as a player in the development and progression of atherosclerotic lesions.

\section{CELL APOPTOSIS AND CELL SURVIVAL}

As stated above, intracellular visfatin/PBEF/Nampt is acknowledged to play a central role in cell maturation and survival. Similarly, extracellular visfatin/PBEF/Nampt exerts anti-apoptotic effects and promotes cell survival. Thus, it has been demonstrated that visfatin/ $\mathrm{PBEF} / \mathrm{Nampt}$ attenuates cell apoptosis induced by hydrogen peroxide in both human endothelial cells (Adya et al., 2008a) and rat vascular smooth muscle cells in vitro (Wang et al., 2009). Importantly, visfatin/PBEF/Nampt promotes the survival of macrophages subjected to endoplasmic reticulum stress, a process associated to obesity and obesity-related complications (Li et al., 2008). Such an effect of visfatin/PBEF/Nampt is not related to Nampt activity, but rather involves the release of interleukin (IL)- 6 by macrophages that in turn acts in a paracrine and/or autocrine manner to activate intracellular STAT3 signaling (Li et al., 2008). These data indicate that visfatin/PBEF/Nampt may affect the balance between macrophage survival and death in obesity. By promoting macrophage survival, visfatin/PBEF/Nampt may contribute, not only to adipose tissue inflammation, but also to vascular inflammation and the progression of atherosclerotic lesions, as will be discussed below.

\section{VASCULAR TONE AND REACTIVITY}

The scarce reports available to date exploring the potential direct actions of visfatin/PBEF/Nampt on the regulation of the vascular tone are contradictory. On one hand, visfatin/PBEF/Nampt has been claimed to stimulate the expression and activity of eNOS in endothelial cells, resulting in enhanced NO production and cyclic GMP formation in human umbilical vein and coronary endothelial cells in vitro (Lovren et al., 2009). By favoring NO release, visfatin/PBEF/Nampt 


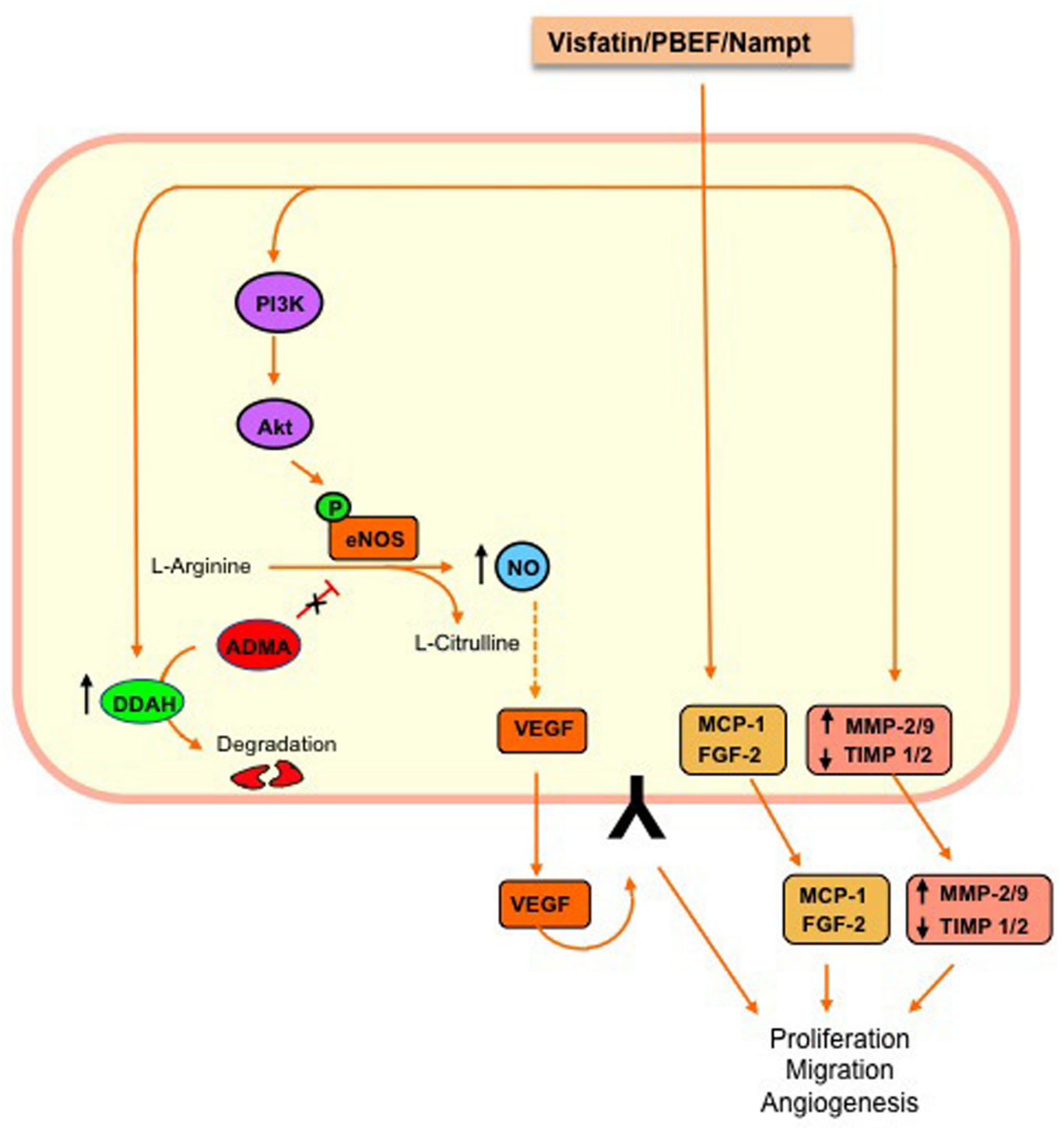

FIGURE 3 | Main signaling pathways that mediate visfatin/PBEF/ Nampt-induced endothelial cell proliferation and migration. Visfatin/PBEF/ Nampt promotes vascular endothelial growth factor (VEGF) synthesis and secretion and up-regulates the expression of the VEGF receptor 2. Visfatin/ PBEF/Nampt favors the endothelial production of nitric oxide (NO), a promoter of VEGF release, by activating endothelial nitric oxide synthase (eNOS) via the $\mathrm{PI}$ KK/Akt pathway. NO production is further enhanced through the activation of dimethylarginine dimethylaminohydrolase (DDAH), which degrades the eNOS inhibitor asymmetric dimethylarginine (ADMA). Additionally, visfatin/PBEF/ Nampt triggers the endothelial production of other pro-angiogenic molecules, such as monocyte chemoatractant protein (MCP)-1 and fibroblast growth factor (FGF)-2. On the other hand, visfatin/PBEF/Nampt enhances the levels and activation of matrix metalloproteinases (MMP)-2/9 while decreasing the levels of tissue inhibitor of metalloproteinases (TIMP)-1/2. might exert a vasorelaxant effect. Accordingly, it has been shown that the addition of visfatin/PBEF/Nampt to an organ bath results in the relaxation of isolated rat aortas pre-contracted with noradrenaline, through a mechanism that is independent of the insulin receptor (Yamawaki et al., 2009).

Nevertheless, Wang et al. (2009) could not demonstrate any contribution of visfatin/PBEF/Nampt on the regulation of the vascular tone exerted by perivascular fat on rat aortas. Furthermore, a growing bulk of evidence shows a positive association between circulating visfatin/PBEF/Nampt levels and endothelial dysfunction, assessed by brachial artery flow-mediated dilation (FMD) in different clinical conditions, including type 2 diabetes mellitus (Takebayashi et al., 2007) and chronic kidney disease (Yilmaz et al., 2008). Conversely, the normalization of endothelial function following a renal transplantation is associated with a reduction in the circulating levels of visfatin/PBEF/Nampt (Yilmaz et al., 2009). These data suggest that, besides representing a potential biomarker for endothelial dysfunction and vascular damage, visfatin/PBEF/ Nampt might play a direct role in impairing vascular relaxation. Additional studies using both in vivo and in vitro approaches are therefore needed to clarify the capacity of visfatin/PBEF/Nampt to directly regulate the vascular tone. 


\section{VASCULAR INFLAMMATION AND ATHEROSCLEROSIS}

In the last years, a large number of reports have highlighted the proinflammatory properties of visfatin/PBEF/Nampt on a wide range of cell types and tissues. As stated in the Introduction, PBEF was first identified as an immunomodulatory cytokine that synergizes with interleukin 7 (IL-7) and stem cell factor (SCF) to promote pre-B cell colony formation (Samal et al., 1994). Visfatin/PBEF/Nampt is now being regarded as a marker of inflammation. Serum visfatin/ $\mathrm{PBEF} / \mathrm{Nampt}$ levels have been positively correlated with circulating inflammatory markers, such as IL-6, CRP and MCP-1 (Oki et al., 2007; Kato et al., 2009; Liu et al., 2009a). Indeed, visfatin/PBEF/ Nampt levels are positively associated to a series of inflammatory conditions, including osteoarthritis, acute lung injury, inflammatory bowel disease and Crohn's disease, gynecological disorders, such as infection-induced pre-term birth, sepsis, or psoriasis (Moschen et al., 2010).

In the context of cardiovascular diseases, visfatin/PBEF/Nampt has been proposed as a marker of atherosclerosis, a clinical condition characterized by low-grade chronic inflammation (Liu et al., 2009a; Kadoglou et al., 2010). Other studies, however, suggest that visfatin/PBEF/NAMPT may not directly reflect atherosclerotic changes, but rather the global inflammatory status in cardiovascular and renal patients (Kato et al., 2009). Importantly, a positive correlation has been found between the expression of visfatin/ $\mathrm{PBEF} / \mathrm{Nampt}$ in the periaortic and pericoronary fat and coronary atherosclerosis (Spiroglou et al., 2010), highlighting that perivascular visfatin/PBEF/Nampt might play an important paracrine role in the development of atherosclerotic lesions.

In this context, a growing bulk of evidence indicates that visfatin/PBEF/Nampt can directly promote vascular inflammation by activating different cell types including endothelial cells, vascular smooth muscle cells, or monocytes/macrophages. Thus, in cultured human umbilical vein endothelial cells, visfatin/PBEF/ Nampt activates the inflammation-related transcription factor NF-KB (Kim et al., 2008; Adya et al., 2009; Lee et al., 2009) and promotes the expression of cell adhesion molecules, including ICAM-1, VCAM-1, or E-selectin (Kim et al., 2008; Lee et al., 2009), as key molecules implicated in leukocyte recruitment and early pro-atherosclerotic events (Galkina and Ley, 2007). Visfatin/ PBEF/Nampt further promotes the endothelial release of several cytokines and chemokines by endothelial cells, including interleukin (IL)-6, IL-8 or MCP-1 and its putative receptor CCR2 (Lee et al., 2009; Liu et al., 2009b), and thus promotes the adhesion of human THP-1 monocytes to endothelial cells (Liu et al., 2009b). The MAPK ERK 1/2 and p38, as well as PI3K and the intracellular generation of reactive oxygen species, have been involved in endothelial cell inflammation induced by visfatin/PBEF/Nampt (Kim et al., 2008; Liu et al., 2009b).

In human vascular smooth muscle cell cultures, visfatin/PBEF/ Nampt consecutively activates ERK $1 / 2$ and NF- $\kappa B$, that in turn stimulates the expression of inducible nitric oxide synthase (iNOS) (Romacho et al., 2009), a NO- and peroxynitrite-forming pro-inflammatory enzyme playing a key role in vascular damage and endothelial dysfunction (Pacher et al., 2005). The pro-inflammatory action of visfatin/PBEF/Nampt in human vascular smooth muscle cells is not mediated by the insulin receptor, but rather relies on Nampt intrinsic activity. Accordingly, the pro-inflammatory action of visfatin/PBEF/Nampt is prevented by the pharmacological Nampt inhibitor APO866 and mimicked by NMN, the final product of the reaction catalyzed by Nampt (Romacho et al., 2009).

Visfatin/PBEF/Nampt can also contribute to vascular inflammation through its direct actions on monocytes. Hence, visfatin/PBEF/ Nampt promotes the synthesis and release of pro-inflammatory cytokines, such as tumor necrosis factor (TNF)- $\alpha$ and IL-8, by peripheral mononuclear cells (Dahl et al., 2007). As stated above, visfatin/PBEF/Nampt promotes macrophage survival (Li et al., 2008), which may help perpetuating vascular inflammation.

Matrix metalloproteinases are pivotal enzymes mediating the degradation of extracellular matrix and facilitating atherosclerotic plaque fragility and disruption. In endothelial cells (Adya et al., 2008b), but also in monocytes (Dahl et al., 2007), visfatin/ PBEF/Nampt induces the expression and activity of MMP-2 and MMP-9. Interestingly, an enhanced visfatin/PBEF/Nampt content has been detected in human unstable versus stable carotid plaque samples (Dahl et al., 2007). Furthermore, a positive association between visfatin/PBEF/Nampt and unstable atherosclerotic lesions is also found in patients with coronary artery disease (CAD) and acute myocardial infarction, with a strong visfatin/PBEF/Nampt immunostaining observed in lipid-rich regions at the site of plaque rupture (Dahl et al., 2007). Immunoreactive visfatin/PBEF/Nampt has also been detected in vascular smooth muscle cells within the atherosclerotic plaque (Spiroglou et al., 2010). Taken together, these findings suggest that visfatin/PBEF/Nampt can be a relevant molecule in plaque weakening.

Through its multiple actions promoting cytokine and chemokine secretion, macrophage survival, leukocyte recruitment by endothelial cells, vascular smooth muscle and endothelial cell inflammation and matrix degradation, visfatin/PBEF/Nampt, either circulating or locally synthesized, may contribute to the development of atherosclerotic lesions and plaque vulnerability and rupture. Visfatin/PBEF/Nampt is therefore gaining relevance as a potential therapeutic target to interfere with the inflammatory response associated to obesity-related complications, especially to atherosclerosis.

\section{CARDIAC ACTIONS}

To date, only few reports have studied the direct effects of visfatin/ PBEF/Nampt on cardiac function, showing apparently opposite results. On one side, visfatin/PBEF/Nampt may be involved in the pathogenesis of myocardial fibrosis; on the other, visfatin/PBEF/ Nampt has been claimed to possess potential cardioprotective properties.

The proliferation of cardiac fibroblasts and the excessive accumulation of extracellular matrix are at the basis of myocardial fibrosis. In vitro studies have recently shown that visfatin/PBEF/ Nampt promotes DNA synthesis and proliferation of cultured cardiac fibroblasts (Yu et al., 2010). The expression and protein levels of procollagen I and II are also elevated in cardiac fibroblasts stimulated with visfatin/PBEF/Nampt, which ultimately leads to enhanced type I and III collagen release (Yu et al., 2010). The stimulation of cardiac fibroblasts by visfatin/PBEF/Nampt involves the activation of Akt/PKB and the MAP kinases p38 and JNK, but not ERK 1/2 (Yu et al., 2010). Taking into account that visfatin/ $\mathrm{PBEF} / \mathrm{Nampt}$ is expressed in periadventitial and apical epicardial 
adipose tissue (Spiroglou et al., 2010), both locally produced and circulating visfatin/PBEF/Nampt could play a detrimental role in promoting myocardial fibrosis.

Conversely, visfatin/PBEF/Nampt could exert direct cardioprotective properties in a clinical condition, like myocardial reperfusion. Hence, Lim et al. (2008) have shown that, in a mouse model of ischemia-reperfusion, the administration of an intravenous bolus of visfatin/PBEF/Nampt at the time of reperfusion reduced the infarct size by around 20\% (Lim et al., 2008). In vitro, murine cardiomyocytes submitted to hypoxia and reoxygenation, visfatin/ $\mathrm{PBEF} /$ Nampt administered at the time of reoxygenation, exhibit significantly diminished cell death delayed, due at least in part to a delayed opening of the mitochondrial permeability transition pore (mPTP) by oxidative stress. The opening of mTPT, a non-specific mitochondrial channel, in the first minutes of reperfusion appears is a critical determinant of cardiomyocyte death (Hausenloy and Yellon, 2003). The mechanism by which visfatin/PBEF/Nampt protects cardiomyocytes from ischemic damage remains unveiled. It has nevertheless been proposed that exogenous visfatin/PBEF/ Nampt may enhance intracellular NAD+ levels through Nampt activity, conferring a higher resistance of cardiomyocytes to oxidant stress. Further studies are required to determine whether visfatin/ $\mathrm{PBEF} /$ Nampt can represent a valuable pharmacological tool to treat cardiac damage derived from ischemia-reperfusion.

\section{CONCLUSIONS AND PERSPECTIVES}

Five years ago, visfatin/PBEF/Nampt was identified as a novel adipocytokine with potential beneficial effects in the context of metabolic disorders, due to its insulin-mimetic and

\section{REFERENCES}

Adya, R., Tan, B. K., Punn, A., Chen, J., and Randeva, H. S. (2008a). Visfatin induces human endothelial VEGF and MMP-2/9 production via MAPK and PI3K/Akt signalling pathways: novel insights into visfatin-induced angiogenesis. Cardiovasc. Res. 78, 356-365.

Adya, R., Tan, B. K., Chen, J., and Randeva, H. S. (2008b). Nuclear factor-kappaB induction by visfatin in human vascular endothelial cells: its role in MMP-2/9 production and activation. Diabetes Care 31, 758-760.

Adya, R., Tan, B. K., Chen, J., and Randeva, H. S. (2009). Pre-B cell colony enhancing factor $(\mathrm{PBEF}) / \mathrm{visfatin}$ induces secretion of MCP-1 in human endothelial cells: role in visfatininduced angiogenesis. Atherosclerosis 205, 113-119.

Arner, P. (2006). Visfatin-a true or false trail to type 2 diabetes mellitus. J. Clin. Endocrinol. Metab. 91, 28-30.

Bae, Y. H., Bae, M. K., Kim, S. R., Lee, J. H., Wee, H. J., and Bae, S. K. (2009). Upregulation of fibroblast growth factor- 2 by visfatin that promotes endothelial angiogenesis. Biochem. Biophys. Res. Commun. 379, 206-211.
Bi, T. Q., and Che, X. M. (2010). Nampt/ $\mathrm{PBEF} /$ visfatin and cancer. Cancer Biol. Ther. 10, 119-125.

Dahl, T. B., Yndestad, A., Skjelland, M., Øie, E., Dahl,A., Michelsen, A., Damås, J. K., Tunheim, S.H., Ueland, T., Smith, C., Bendz, B., Tonstad, S., Gullestad, L., Frøland, S. S., Krohg-Sørensen, K., Russell, D., Aukrust, P., and Halvorsen, B. (2007). Increased expression of visfatin in macrophages of human unstable carotid and coronary atherosclerosis: possible role in inflammation and plaque destabilization. Circulation 115, 972-980.

Fiedler, L. R., Bachetti, T., Leiper, J., Zachary, I., Chen, L., Renné, T., and Wojciak-Stothard, B. (2009). The ADMA/DDAH pathway regulates VEGF-mediated angiogenesis. Arterioscler. Thromb. Vasc. Biol. 29, 2117-2124.

Filippatos, T. D., Randeva, H. S., Derdemezis, C. S., Elisaf, M. S., and Mikhailidis, D. P. (2010). Visfatin/ $\mathrm{PBEF}$ and atherosclerosis-related diseases. Curr. Vasc. Pharmacol. 8, 12-28.

Formentini, L., Moroni, F., and Chiarugi, A. (2009). Detection andpharmacological modulation of nicotinamide mononucleotide (NMN) in vitro

glucose-lowering properties. Nevertheless, growing evidence has since then unveiled that visfatin/PBEF/Nampt may rather be a biomarker of inflammation and endothelial damage in metabolic diseases. Several reports have now demonstrated that visfatin/PBEF/Nampt may exert direct deleterious actions on the cardiovascular system, including cell proliferation, monocyte/ macrophage activation and recruitment, vascular inflammation and extracellular matrix remodeling, all of which can contribute to the development of atherosclerotic lesions. In this context, the pharmacological blockade of the cardiovascular actions on the visfatin/PBEF/Nampt might represent a novel therapeutic approach to prevent and treat cardio-metabolic complications. The blockade of visfatin/PBEF/Nampt-mediated angiogenesis might also be useful in treating but pathologies implying excessive neovascularization. On the other hand, not all the cardiovascular actions of visfatin/PBEF/Nampt are detrimental, since the administration of visfatin/PBEF/Nampt has shown beneficial effects in clinical conditions, such as peripheral limb ischemia or myocardial ischemia-reperfusion, where it may become a beneficial pharmacological tool. Further research, using both in vitro and in vivo approaches, is required to evaluate the real value of visfatin/PBEF/Nampt as a pharmacological target in the cardiovascular system.

\section{ACKNOWLEDGMENTS}

Supported by grants from Ministerio de Ciencia e Innovación (SAF2008-01291, SAF2008-00942, and FIS PI061779). Tania Romacho is the recipient of a FPU fellowship from Ministerio de Educación.

and in vivo. Biochem. Pharmacol. 77, 1612-1620.

Fukuhara,A., Matsuda, M., Nishizawa, M., Segawa, K., Tanaka, M., Kishimoto, K., Matsuki,Y., Murakami, M., Ichisaka, T., Murakami, H., Watanabe, E., Takagi, T., Akiyoshi, M., Ohtsubo, T., Kihara, S., Yamashita, S., Makishima, M., Funahashi, T., Yamanaka, S.,Hiramatsu, R., Matsuzawa, Y., and Shimomura, I. (2005). Visfatin: a protein secreted by visceral fat that mimics the effects of insulin. Science 307, 426-430.

Fukuhara,A., Matsuda, M., Nishizawa, M., Segawa, K., Tanaka, M., Kishimoto, K. Matsuki, Y., Murakami, M., Ichisaka, T., Murakami, H., Watanabe, E., Takagi, T., Akiyoshi, M., Ohtsubo, T., Kihara, S., Yamashita, S., Makishima, M., Funahashi, T., Yamanaka, S., Hiramatsu, R., Matsuzawa, Y., and Shimomura, I. (2007). Retraction. Science 318, 565.

Galkina, E., and Ley, K. (2007). Vascular adhesion molecules in atherosclerosis. Arterioscler. Thromb. Vasc. Biol. 27, 2292-2301.

Garten, A., Petzold, S., Körner, A., Imai, S., and Kiess, W. (2009). Nampt: linking NAD biology, metabolism and cancer. Trends Endocrinol. Metab. 20, 130-138.
Guzik, T. J., Mangalat, D., and Korbut, R. (2006). Adipocytokines. Novel link between inflammation and vascular function? J. Physiol. Pharmacol. 57, 505-528.

Hausenloy, D. J., and Yellon, D. M. (2003). The mitochondrial permeability transition pore: its fundamental role in mediating cell death during ischaemia and reperfusion. J. Mol. Cell Cardiol. 35, 339-341.

Ho, C., van der Veer, E., Akawi, O., and Pickering, J. G. (2009). Sirtl markedly extends replicative lifespan if the nad+ salvage pathway is enhanced. FEBS Lett. 583, 3081-3085.

Kadoglou,N.P.,Sailer,N.,Moumtzouoglou, A., Kapelouzou, A., Tsanikidis, H., Vitta, I., Karkos, C., Karayannacos, P. E., Gerasimidis, T., and Liapis, C. D. (2010).Visfatin (NAMPT) and ghrelin as novel markers of carotid atherosclerosis in patients with type 2 diabetes. Exp. Clin. Endocrinol. Diabetes 118, 75-80.

Karastergiou, K., and Mohamed-Ali, V. (2010). The autocrine and paracrine roles of adipokines. Mol. Cell Endocrinol. 318, 69-78.

Kato, A., Odamaki, M., Ishida, J., and Hishida, A. (2009). Relationship between serum pre-b cell 
colony-enhancing factor/visfatin and atherosclerotic parameters in chronic hemodialysis patients. Am. J. Nephrol. 29, 31-35.

Kim, J. Y., Bae, Y. H., Bae, M. K., Kim, S. R., Park, H. J., Wee, H. J., and Bae, S. K. (2009). Visfatin through STAT3 activation enhances IL-6 expression that promotes endothelial angiogenesis. Biochim. Biophys. Acta 1793, 1759-1767.

Kim, S. R., Bae, Y. H., Bae, S. K., Choi, K. S., Yoon, K. H., Koo, T. H., Jang, H. O., Yun, I., Kim, K. W., Kwon, Y. G., Yoo, M. A., and Bae, M. K. (2008). Visfatin enhances ICAM-1 and VCAM-1 expression through ros-dependent NF-kappaB activation in endothelial cells. Biochim. Biophys. Acta 1783, 886-895.

Kim, S. R., Bae, S. K., Choi, K. S., Park, S.Y., Jun, H. O., Lee, J.Y., Jang, H. O., Yun, I., Yoon, K. H., Kim, Y. J., Yoo, M. A., Kim, K. W., and Bae, M. K. (2007). Visfatin promotes angiogenesis by activation of extracellular signal-regulated kinase 1/2. Biochem. Biophys. Res. Commun. 357, 150-156.

Lau, D. C., Dhillon, B., Yan, H., Szmitko, P. E., and Verma, S. (2005). Adipokines: molecular links between obesity and atherosclerosis. Am. J. Physiol. Heart Circ. Physiol. 288, 2031-2041.

Lee, W. J., Wu, C. S., Lin, H., Lee, I. T., Wu, C. M., Tseng, J. J., Chou, M. M., and Sheu, W. H. (2009). Visfatin-induced expression of inflammatory mediators in human endothelial cells through the NF- $\kappa \mathrm{B}$ pathway. Int. J. Obes. (Lond). 33, 465-472.

Li, Y., Zhang, Y., Dorweiler, B., Cui, D., Wang, T., Woo, C. W., Brunkan, C. S., Wolberger, C., Imai, S., and Tabas, I. (2008). Extracellular nampt promotes macrophage survival via a nonenzymatic interleukin-6/STAT3 signaling mechanism. J. Biol. Chem. 283, 34833-34843.

Lim, S. Y., Davidson, S. M., Paramanathan, A. J., Smith, C. C., Yellon, D. M., and Hausenloy, D. J. (2008). The novel adipocytokine visfatin exerts direct cardioprotective effects. J. Cell Mol. Med. 12, 1395-1403.

Liu, S. W., Qiao, S. B., Yuan, J. S., and Liu, D. Q. (2009a). Association of plasma visfatin levels with inflammation, atherosclerosis and acute coronary syndromes (ACS) in humans. Clin. Endocrinol. (Oxf). 71, 202-207.

Liu, S. W., Qiao, S. B., Yuan, J. S., and Liu, D. Q. (2009b). Visfatin stimulates production of monocyte chemotactic protein- 1 and interleukin-6 in human vein umbilical endothelial cells. Horm. Metab. Res. 41, 281-286.

Lovren, F., Pan, Y., Shukla, P. C., Quan, A., Teoh, H., Szmitko, P. E., Peterson, M. D., Gupta, M., Al-Omran, M., and Verma, S. (2009). Visfatin activates eNOS via Akt and MAP Kinases and improves endothelial cell function and angiogenesis in vitro and in vivo: translational implications for atherosclerosis. Am. J. Physiol. Endocrinol. Metab. 296, 1440-1449.

Moschen, A. R., Gerner, R. R., and Tilg, H. (2010). Pre-B cell colony enhancing factor/NAMPT/visfatin in inflammation and obesity-related disorders. Curr. Pharm. Des. 16, 1913-1920.

Oki, K., Yamane, K., Kamei, N., Nojima, H., and Kohno, N. (2007). Circulating visfatin level is correlated with inflammation, but not with insulin resistance. Clin. Endocrinol. (Oxf). 67, 796-800.

Pacher, P., Obrosova, I. G., Mabley, J. G., and Szabo, C. (2005). Role of nitrosative stress and peroxynitrite in the pathogenesis of diabetic complications. Emerging new therapeutical strategies. Curr. Med. Chem. 12, 267-275.

Pogrebniak, A., Schemainda, I., Azzam, K., Pelka-Fleischer, R., Nüssler, V., and Hasmann, M. (2006). Chemopotentiating effects of a novel NAD biosynthesis inhibitor, FK866, in combination with antineoplastic agents. Eur. J. Med. Res. 11, 313-321.

Ravaud, A., Cerny, T., Terret, C., Wanders, J., Bui, B. N., Hess, D., Droz, J. P., Fumoleau, P., and Twelves, C. (2005). Phase I study and pharmakokinetic of CHS-828, a guanidino-containing compound, administered orally as a single dose every 3 weeks in solid tumours: an ECSG/EORTC study. Eur. J. Cancer 41, 702-707.

Revollo, J. R., Grimm, A. A., and Imai, S. (2004). The NAD biosynthesis pathway mediated by nicotinamide phosphoribosyltransferase regulates Sir2 activity in mammalian cells. J. Biol. Chem. 279, 50754-50763.

Revollo, J. R., Körner, A., Mills, K. F., Satoh, A., Wang, T., Garten, A., Dasgupta, B., Sasaki, Y., Wolberger, C., Townsend, R. R., Milbrandt, J., Kiess, W., and Imai, S. (2007). Nampt/PBEF/Visfatin regulates insulin secretion in beta cells as a systemic NAD biosynthetic enzyme. Cell Metab. 6, 363-375.

Romacho, T., Azcutia, V., Vázquez-Bella, M., Matesanz, N., Cercas, E., Nevado, J., Carraro, R., Rodríguez-Mañas, L., Sánchez-Ferrer, C. F., and Peiró, C. (2009). Extracellular PBEF/NAMPT/ visfatin activates pro-inflammatory signalling in human vascular smooth muscle cells through nicotinamide phosphoribosyltransferase activity. Diabetologia 52, 2455-2463.

Rongvaux, A., Shea, R. J., Mulks, M. H., Gigot, D., Urbain, J., Leo, O., and Andris, F. (2002). Pre-B-cell colonyenhancing factor, whose expression is up-regulated in activated lymphocytes, is a nicotinamide phosphoribosyltransferase, a cytosolic enzyme involved in NAD biosynthesis. Eur. J. Immunol. 32, 3225-3234.

Samal, B., Sun, Y., Stearns, G., Xie, C., Suggs, S., and McNiece, I. (1994). Cloning and characterization of the cdna encoding a novel human pre-Bcell colony-enhancing factor. Mol. Cell Biol. 14, 1431-1437.

Sandeep, S., Velmurugan, K., Deepa, R., and Mohan, V. (2007). Serum visfatin in relation to visceral fat, obesity, and type 2 diabetes mellitus in Asian Indians. Metabolism 56, 565-570.

Spiroglou, S. G., Kostopoulos, C. G., Varakis, J. N., and Papadaki, H. H. (2010). Adipokines in periaortic and epicardial adipose tissue: differential expression and relation to atherosclerosis. J. Atheroscler. Thromb. 17, 115-130.

Stephens, J. M., and Vidal-Puig, A. J. (2006). An update on visfatin/pre-b cell colony-enhancing factor, an ubiquitously expressed, illusive cytokine that is regulated in obesity. Curr. Opin. Lipidol. 17, 128-131.

Takebayashi, K., Suetsugu, M., Wakabayashi, S., Aso, Y., and Inukai, T. (2007). Association between plasma visfatin and vascular endothelial function in patients with type 2 diabetes mellitus. Metabolism 56, 451-458.

van der Veer, E., Ho, C., O'Neil, C., Barbosa, N., Scott, R., Cregan, S. P., and Pickering, J. G. (2007). Extension of human cell lifespan by nicotinamide phosphoribosyltransferase. J. Biol. Chem. 282, 10841-10845.

van der Veer, E., Nong, Z., O’Neil, C., Urquhart, B., Freeman, D., and Pickering, J.G. (2005).Pre-b-cell colony-enhancing factor regulates $\mathrm{NAD}+$-dependent protein deacetylase activity and promotes vascular smooth muscle cell maturation. Circ. Res. 97, 25-34.

Wang, P., Xu, T. Y., Guan, Y. F., Su, D. F., Fan, G. R., and Miao, C. Y. (2009). Perivascular adipose tissue-derived visfatin is a vascular smooth muscle cell growth factor: role of nicotinamide mononucleotide. Cardiovasc. Res. 81, 370-380.

Xiao, J., Xiao, Z. J., Liu, Z. G., Gong, H. Y., Yuan, Q., Wang, S., Li, Y. J., and Jiang,
D. J. (2009). Involvement of dimethylarginine dimethylaminohydrolase-2 in visfatin-enhanced angiogenic function of endothelial cells. Diabetes Metab. Res. Rev. 25, 242-249.

Yamawaki, H., Hara, N., Okada, M., and Hara, Y. (2009). Visfatin causes endothelium-dependent relaxation in isolated blood vessels. Biochem. Biophys. Res. Commun. 383, 503-508.

Yilmaz, M. I., Saglam, M., Carrero, J. J., Qureshi, A. R., Caglar, K., Eyileten, T., Sonmez, A., Cakir, E., Yenicesu, M., Lindholm, B., Stenvinkel, P., and Axelsson, J. (2008). Serum visfatin concentration and endothelial dysfunction in chronic kidney disease. Nephrol. Dial. Transplant. 23, 959-965.

Yilmaz, M. I., Saglam, M., Carrero, J. J., Qureshi, A. R., Caglar, K., Eyileten, T., Sonmez, A., Oguz, Y., Aslan, I., Vural, A., Yenicesu, M., Stenvinkel, P., Lindholm, B., and Axelsson, J. (2009). Normalization of endothelial dysfunction following renal transplantation is accompanied by a reduction of circulating visfatin/nampt. A novel marker of endothelial damage? Clin. Transplant. 23, 241-248.

Yu, X. Y., Qiao, S. B., Guan, H. S., Liu, S. W., and Meng, X. M. (2010). Effects of visfatin on proliferation and collagen synthesis in rat cardiac fibroblasts. Horm. Metab. Res. 42, 507-513.

Conflict of Interest Statement: The authors declare that the research was conducted in the absence of any commercial or financial relationships that could be construed as a potential conflict of interest.

Received: 01 August 2010; paper pending published: 19 August 2010; accepted: 25 October 2010; published online: 23 November 2010.

Citation: Peiró C, Romacho T, Carraro R and Sánchez-Ferrer CF (2010) Visfatin/ PBEF/Nampt: a new cardiovascular target? Front. Pharmacol. 1:135. doi: 10.3389/ fphar.2010.00135

This article was submitted to Frontiers in Cardiovascular and Smooth Muscle Pharmacology, a specialty of Frontiers in Pharmacology.

Copyright $\odot 2010$ Peiró, Romacho, Carraro and Sánchez-Ferrer. This is an open-access article subject to an exclusive license agreement between the authors and the Frontiers Research Foundation, which permits unrestricted use, distribution, and reproduction in any medium, provided the original authors and source are credited. 